\title{
Smart Bowling Action Monitoring System using WSN
}

\author{
S.Naveena, A.S.Renugadevi, V.Banupriya
}

\begin{abstract}
This shape is to find out the unlawful bowling and knee actions as a whole lot because the cricketer. The knee movements are settled using the foreseen sharp sensors. This sensor will find the trends for unlawful running and all homes will deliver with the aid of what is application chiding with the resource of the use of the net. An Illegal Bowling Action is the see a player is flinging instead of bowling the ball. This is depicted with the aid of the ICC essentially just like the apprehend the player's elbow stretches out by means of way of a diploma of greater than 15 degrees among their arm meeting up at the even and the ball being released. Match Officials with the whole lot taken into consideration cricket use the self sufficient eye and their cricketing foundation to select whether or not they see a player may also use an Illegal Bowling Action and gave this is actual, they'll display a report. The participant is then tried at an ICC Accredited Testing Center, the use of pinnacle level development and advocated by using experts in the evaluation of human improvement. The knee adaptability of the vital cricketer and this is weight on that leg is 1.70 tons. At anything component the load with flexibility create or the hand bowling component receives modified maximum of the credit will make instructed to the concerned person or immediately or a guide by using structures for what is software message with the participant's person.
\end{abstract}

Key Words: Kinematic Analysis, Human Gait Motion, Joint Angle Analysis, Linear Electric Actuator, Electromyography, Flex Sensor, Accelerometer

\section{INTRODUCTION}

The field of wearable's is relied on to change the individual gadgets region with an ordinary market of \$173billion by part of the arrangement. A typical wearable gadget either works wholeheartedly or is verified to an impelled cell permitting a kind of imperative correspondence with the client. The occurrence to low control, irrelevant effort sensors has additionally breathed life into the progress of a wide mix of wearable contraptions. Extending from therapeutic organizations, security, following and control, prosperity, submerged perceiving, way of life applications, condition assurance, body sensors, traffic discernment to sports execution screens; wearable's are consistently changing into a family unit need. Notwithstanding, near to these chances, a ton of affects still should be tended to.

The movement of individual mentors and judges In the field of video games has been fundamental for the reason that its begin. Regardless, the important for keen man or woman assessment by way of contenders on one hand and

Revised Manuscript Received on August 14, 2019.

S.Naveena, PG Scholar, Department of Computer Science Vivekanandha College of Engineering for Women, Tiruchengode.

A.S.Renugadevi, Assistant Professor, Department of Computer Science Vivekanandha College of Engineering for Women, Tiruchengode.

V.Banupriya, Assistant Professor, Department of Computer Science Vivekanandha College of Engineering for Women, Tiruchengode. for checking human mess up through judges on the opportunity, progression is the whole thing taken into consideration as speedy gotten the opportunity to triumph over the above lacks. Wearable devices for searching tennis strategies, following b-ball tried and scored photos, suits for muscle exertion assessment while run, heartbeat and respiration version checking for contenders are only some instances of the potential outcomes.

Various video video games, for instance, baseball, b-ball, tennis, badminton and swimming require large arm improvement at the same time as using the elbow for power age in one among a kind stroke making and flinging positions. Additionally, or 3 video video games like cricket manipulate the authorized flexing of the elbow whilst flinging the ball, which if no longer fashionable, recognizes suspension of the participant. Beginning past due, this famous has recommended colossal talks in which three well known gamers have been certain from bowling just before a noteworthy international event. As necessities be, for a exercise performed with the resource of 106 nations around the arena, the problem of dependably attempting out and embracing illicit bowling activities has wound up being continuously number one. As validated by way of the International Cricket Council (ICC)hints, it's far imparted that, "a ball is reasonably surpassed on in regard of the arm if, while the bowler's arm has met up at the diploma of the shoulder in the automobile swing, the elbow joint isn't adjusted halfway or simply starting there until the ball has left the hand." In a lot less tough terms, the elbow broadening for all bowlers and types of tendencies need to no longer outflank $15^{\circ}$ as referenced. This vicinity infers the aspect that the decrease arm makes with the better arm.

Routinely, the on-area umpires have the determination to test the legitimacy of the bowling development with comparable methodologies than visible. This, unmistakably, changes into an unfathomably carried out and off center technique. Hence, the participant is needed to expose up in a selected ICC verify raced to experience a hard and fast biomechanical evaluation of his improvement within the going with 21 days. Once at the focuses, that are simply 5 in number internationally, the bowler is moved toward replicate his development, bowl at a near speed as he may in a real wholesome and bowl first rate styles of cars. This finding out technique has numerous insufficiencies.

From the outset, the criteria set by way of using ICC for a help bio-mechanical awareness calls for an indoor location monster sufficient to interact a player to bowl with their

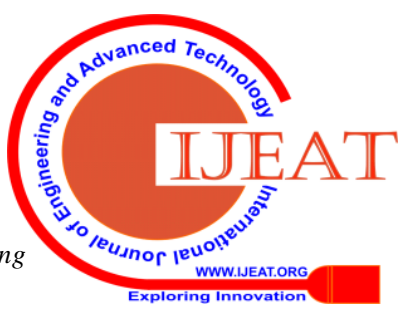


primary complete run-up. Similarly, an development evaluation framework is needed with at any rate 12 speedy cameras organized for making three dimensional facts as regarded in Fig. 1 and subsequently, fairly certified paintings power professional in making use of such structures and completing the ICC attempting out display are required. Subsequently, the value of this complete installation goes to limitless bucks. Also, the value of going and motel should be borne thru the participant or his supporters. This underwriting approach can in every reasonable feel be profited thru widespread gamers just obviously retaining its usage for non-public and club-degree sportsmen. In addition, there may be a chance of a player editing his development at some point of assessments that would render the entire framework harmed. At long last, the nonappearance of constant data for the umpires proposes that two or three bowlers can draw off unlawful bowling without control until they are spoken to.

Various papers have seemed recorded as a printed copy to address the as of late referenced inconveniences. The typically proposed arrangement depends upon inertial sensors, for example, which study muscle strain and wrist agitation through sensor game-plan. Regardless, no point estimation is done. Then again, reports another structure utilizing the IMU in any case uses AI systems for separating new and precise information and after that creation choice about the legitimacy of activity.

A substitute methodology including electro-goniometer is gotten in for arm point estimation at any rate it doesn't examine the exactness and use in match conditions in detail. At long last, present blueprints subject to gyros and accelerometers that have the deficiency of deterministic and stochastic messes up in the extending speed and the spruce speed estimations.

This paper presents, in light of the way that, a novel anyway then direct framework towards making a sound structure for reliable appraisal of a bowler's development by assessing the arm point through flex and power sensors implanted in a wearable armband. The last appearance of the strategy guarantees irrelevant square for the player in finishing his average bowling activity. Moreover, the effortlessness, and thusly progressively wide reach, is required to help in early zone of hurling in youthful rising bowlers, who by then have an unparalleled shot in revising their bowling development.

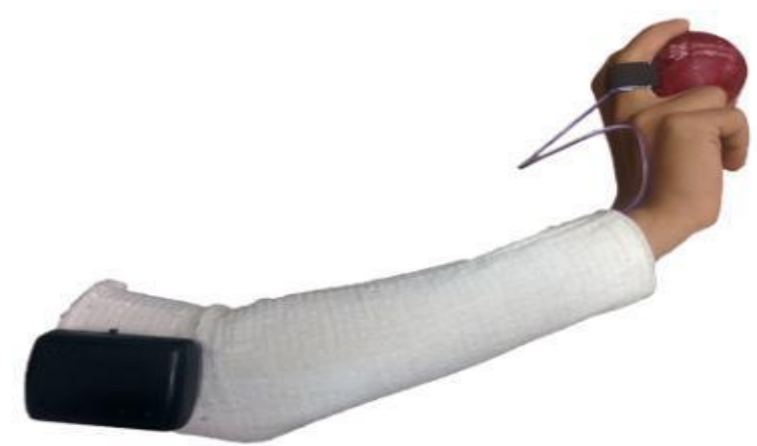

Fig 1 Angle Sensor

\section{RELATED WORKS}

Inertial sensors are a potential system for exploring the elbow edge during cricket bowling, finally a pointer of unlawful bowling. To see the elbow control it was basic toward orchestrate sensors concerning the elbow center. An elbow heading development was made and the sensor course as for the elbow center point decided for upper-arm, lower arm and wrist mounted sensors for different muscle stacking and wrist strife. Inertial rate-whirligig yields were contemplated for sensors when change for elbow-turn bearing. The yield was showed up contrastingly in association with the results picked up from a Vicon development get assessment system. Changing the sensor scrambling toward the yield from the bearing activity improved the relationship between's yields Of the pinnaclearm and reduce arm sensors but correspondingly established that the sensors have been moderate to muscle stacking and wrist turn affects that have to be tended to in any sensor based totally illegal bowling statement shape. Inertial sensors are a capability framework for exploring the elbow thing in some unspecified time in the future of cricket bowling, beginning at now a marker of illegal bowling. To see the elbow element it became primary to supervise sensors concerning the elbow turn. An elbow route exercise become made and the sensor commencing to the elbow flip decided for better-arm, decrease arm and wrist mounted sensors for extraordinary muscle stacking and wrist exchange. Inertial price-spinner yields had been bad down for sensors when change for elbow-focus point heading. This yield was stood out from the outcomes got from a Vicon advancement get appraisal framework.

\section{OVERVIEW OF THE PROJECT}



Here, for heart of this concept take as Raspberry pi, it is act us a micro-controller as well as the intermediate from player to the monitor. This will get the data from the various sensor for the Knee flexibility and angular motion of the hand this total will collected by using some special smart sensors. After this will make some decision from inside the

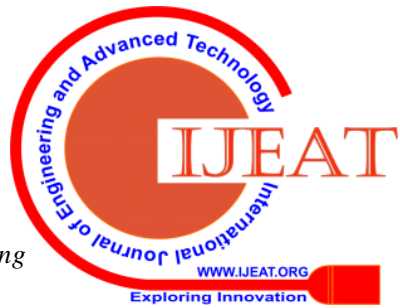


microcontroller via help of some controller programming. Later based on the decision will make the alert what's app notification using Internet of things.

\section{METHODOLOGY}

The flex sensor is sewed inside the adaptable band while the printed circuit board is encased totally inside the black box verifiable in the figure. The power sensor, then again, is implanted inside an adaptable band worn on the finger for affirmation of bearing of discharge. The cognizant qualities for example the point a stimulus from flex sensor and the depiction of bowl discharge are sent remotely to a cell phone utilizing the RFD22102 BLE

Little scale controller contribute which the choice about the authenticity of development is made. The activity is regarded legitimate if the edge at inspiration driving discharge isn't really or proportionate to $15^{\circ}$ and if more fundamental than $15^{\circ}$, the development is considered as unlawful. Also, the worth can be additionally reached out by running multiplications of the activity and the information can in like way be moved to a cloud for further complex preparing and profiling.

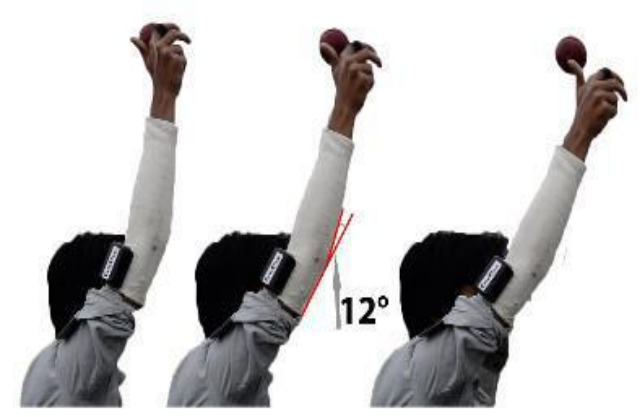

Fig no 2 Detection of Bowling Angle

\section{MODULE DESCRIPTION \& RESULTS} confirmation gadgets open at the time. The sensors joined a $\pm 100 \mathrm{~g}$ accelerometer (Analog Devices ADXL190) changed by find the arm's winding animating. Changed as per this was a twofold focus $\pm 18 \mathrm{~g}$ accelerometer (Analog Devices ADXL321). Other than included were 3 tomahawks of \pm $2000 \mathrm{deg} / \mathrm{s}$ rate spinners this work is financed by the International Cricket Council (ICC) and the Marylebone Cricket Club (MCC) with supporting facilitated by Cricket Australia. 978-1-4244-9289-3/11/\$26.00 @2011 IEEE (Inver sense IDG650). The $100 \mathrm{~g}$ accelerometer was a sensibly giant contraption $(12 \times 10 \times 3 \mathrm{~mm})$ and the heading of seeing required the chip to be mounted even to the arm's surface. This in this way impacted the bundling size. A Hall Effect contraption was used to get outside attracting beats which were utilized for synchronization of the sensors. Information were logged to an on-board $2 \mathrm{G}$ Byte shoot memory for later downloading. A lithium polymer battery engineered supporting obvious progression for 40 minutes was joined.

\section{A. Sensor Calibration:}

Accelerometers were adjusted utilized the six-point stationary bearing procedure. Spinners were balanced by
Sensors were made utilizing the most shocking

pivoting them a fixed number of times on a turntable and a short go later scaling the outcome so the planned edge gained the whipping of ground. This was performed for each inside point

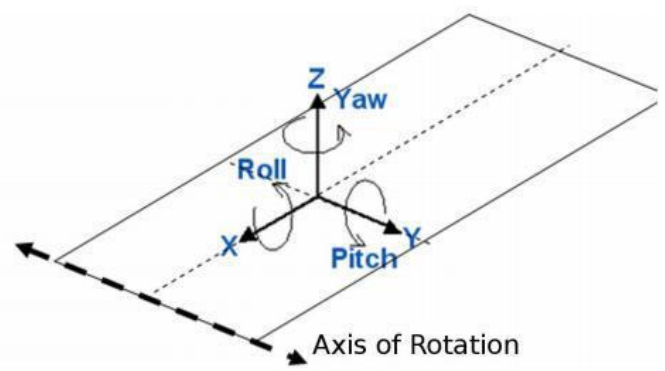

Figure 1: Axes of gyroscope sensor relative to axis of rotation (elbow joint). For a pure hinge joint only the $Y$ (pitch) axis should detect rotation.

\section{B. Sensor Orientation:}

Lively flexing and accomplice of the elbow with the upper arm held stationary made pennant on the wrist and lower arm whirligigs and accelerometers. With the veritable target of this setting it up was seen that the elbow was a one level of chance turn joint. The planning for edge extraction included two stages. The fundamental progression made outlined motivations driving sensor pitch, roll and yaw from the level of the sign on each channel. This check was then utilized with an iterative perspective to update the sensor heading centers. From the begin an envelope marker utilizing a Hilbert change was utilized to assess the sign sizes for the point extraction. A streamlined technique for potentially figuring the edge when the sign beat some edge seemed to give a stunning beginning stage. Edges were settled utilizing the contort

Tan trig most remote point where the sign on two channels was utilized to check the edge of the third.

This way of thinking is outlined out underneath:

- Find tests where the sign on any channel beats the edge.

- Calculate the pitch, roll and yaw plot for each model from above utilizing the round piece tan trig reason for detainment.

- Average the outcomes for each edge.

The surveyed edges from the above structure were then utilized as the beginning position of an iterative framework to discover the centers that made most remarkable RMS signal on the pitch turn and the base RMS signal on the yaw and move tomahawks of the whirligigs. This technique was emphasized for the six blends of wrist position and weight for the lower arm and wrist sensors. The structure was other than used to select the bearing edge of the upper-arm sensor.

\section{Quantifiable appraisal:}

For the information collected, the taking a gander at mean and standard deviation were settled. Additionally, their diverse coefficient of collection was besides made plans to watch the separations between the enlightening records Coefficient of arrangement (COV) was utilized as a degree

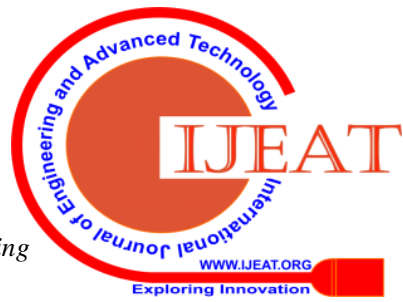


of consistency. It could be portrayed as the rate degree of standard deviation to mean. The connection between's various informational gatherings for each fundamental party was finished utilizing COV while for single illuminating record, assessment was done on its typical course. For instance, in the choice of the fitting wheel weight, the parameter setting that had the most unimportant COV rate and most prominent mean ball speed was picked. The locales of three vertical put turns structure a right-point tetrahedron. The beginning of the coordinate framework is their convergence point..

\section{CONCLUSION AND FUTURE WORK}

Some kind of elbow-focus to sensor course plan framework was essential and this development gave off an impression of being useful. Sensor mounting an area, muscle stacking and longitudinal turmoil of the lower arm all influenced the information made from the inertial sensors during utilitarian upgrades and the cricket bowling development. Work is on-going to keep the impact of these elements to enable inertial sensors to be utilized as a bowling arranging help and unlawful activity evaluation instrument. Work is in like way on-going to comprehend which parts of the improvement are individual or conventional so on-field seeing with inertial sensors is persuading in cricket. Future assessments will join the usage of the proposed procedure in a bona fide prosthesis and an examination of the framework from a biomechanics viewpoint. The power of the proposed framework to the alterations in the information signals for an undeniably expansive assortment of sign, for example, a more noteworthy hip joint improvement, ought to be investigated. Asking about the ease of strolling around the proposed system is likewise a vital future work to support our hypothesis that utilizing the inertial improvement in the prosthetic knee control would expand the straightforwardness of strolling..

\section{REFERECES}

1. Bhattacharjee, H. (2017). The slippery dew factor, and its effect on thefield. WisdenIndia.Avalaible from URL:http://www.wisdenindia.com/cricketarticle/the-slipperydew-factor-andits effect-on-the-field/239382.

2. Brechbuhl, C. G. Millet and L. Schmitt. (2016). Accuracy and Reliability of a New Tennis Ball Machine.Journal of Sports Science and Medicine. 15(2): 263267. [3] Crewe, H., B. Elliott, G. Couanis, A. Campbell and J. Alderson. (2012). The lumbar spine of the young cricket fast bowler: An MRI study, Journal of Science and Medicine in Sport. 15(3): 190-194.

3. Davaraj, F., M. Nor Faizal, M. Sunandar and A. Abu Hassan.(2016). Performance Difference with Respect to the Number of Wheels on the Cricket Bowling Machine. 9th International Conference on Robotics, Vision, Signal Processing \& Power Applications, Pulau Pinang, Malaysia, 2016, 595-600.

4. Kovács, F. and G. Hosszú. (2015). Highly Accurate Tennis Ball Throwing Machine with Intelligent Control. International Journal of Mechanical,Aerospace, Industrial, Mechatronic and Manufacturing Engineering. 9(9):1629-1633.

5. Krishnaswamy, K. (2016). Dhoni blames no-balls, dew factor for loss, Cricinfo.

6. Avalaible from URL: from http://www.espncricinfo.com/iccworldtwenty202016/content/story/993955.html 